\title{
Analytical Engines are Unnecessary in Top-down Partitioning-based Placement*
}

\author{
C. J. ALPERT ${ }^{\mathrm{a}}$, A. E. CALDWELL ${ }^{\mathrm{b}}$, T. F. CHAN $^{\mathrm{c}}$, D. J.-H. HUANG ${ }^{\mathrm{d}}$, \\ A. B. KAHNG ${ }^{\mathrm{b}, \dagger}$, I. L. MARKOV ${ }^{\mathrm{b}}$ and M. S. MOROZ \\ ${ }^{a}$ IBM Austin Research Laboratory, Austin, TX 78758; \\ ${ }^{\mathrm{b}}$ UCLA Computer Science Dept., Los Angeles, CA 90095-1596; \\ ${ }^{\mathrm{c}}$ UCLA Mathematics Dept., Los Angeles, CA 90095-1555; \\ ${ }^{\mathrm{d}}$ Silicon Perspective Corp., Santa Clara, CA 95054; \\ ${ }^{\text {e } U C L A ~ A n d e r s o n ~ G r a d u a t e ~ S c h o o l ~ o f ~ M a n a g e m e n t, ~ L o s ~ A n g e l e s, ~ C A ~} 90095$
}

(Received 7 September 1998; In final form 20 November 1998)

\begin{abstract}
The top-down "quadratic placement" methodology is rooted in such works as $[36,9,32]$ and is reputedly the basis of commercial and in-house VLSI placement tools. This methodology iterates between two basic steps: solving sparse systems of linear equations to achieve a continuous placement solution, and "legalization" of the placement by transportation or partitioning. Our work, which extends [5], studies implementation choices and underlying motivations for the quadratic placement methodology. We first recall some observations from [5], e.g., that (i) Krylov subspace engines for solving sparse linear systems are more effective than traditional successive over-relaxation (SOR) engines [33] and (ii) that correlation convergence criteria can maintain solution quality while using substantially fewer solver iterations. The focus of our investigation is the coupling of numerical solvers to iterative partitioners that is a hallmark of the quadratic placement methodology. We provide evidence that this coupling may have historically been motivated by the pre-1990's weakness of min-cut partitioners, i.e., numerical engines were needed to provide helpful hints to weak min-cut partitioners. In particular, we show that a modern multilevel FM implementation [2] derives no benefit from such coupling. We also show that most insights obtained from study of individual min-cut partitioning instances (within the top-down placement) also hold within the overall context of a complete top-down placer implementation.
\end{abstract}

Keywords: Quadratic placement, multi-level min-cut partitioning, hierarchical top-down placement

\footnotetext{
* This work was supported by a grant from Cadence Design Systems, Inc., and is an extension of work reported in Proc. ACM/ IEEE Design Automation Conference, 1997 [5]. See http://vlsicad.cs.ucla.edu for more information.

${ }^{\dagger}$ Corresponding author.
} 


\section{INTRODUCTION}

In the physical implementation of deep-submicron ICs, placement solution quality is a major determinant of whether timing correctness and routing completion will be achieved. The first-order objective is to place connected modules close together, to reduce total routing and lower bounds on signal delay. This implies a minimum wirelength based placement objective. Because there are many layout iterations (including those between placement and global/detailed routing, performance optimizations, technology mapping and logic synthesis) and because fast (constructive) placement estimation is needed within the floorplanner, an ideal placement tool will offer fast, consistent, and high-quality results. Due to its speed, "global" perspective, and ability to address wirelength-based objectives, the quadratic placement methodology ( $c f$. such antecedents as $[36,14,9,33])$ has been widely adopted in industry.

In this work, we revisit the quadratic placement methodology and develop insights into its effective implementation, particularly in light of recent algorithmic developments for partitioning. Our paper is organized as follows. After defining notation, Section 2 synthesizes a generic model of the quadratic placement methodology. The key elements of our model are: (i) top-down hierarchical placement, and (ii) use of a sparse linear systems solver, coupled with a min-cut iterative (FM-type) partitioner, to obtain any given partition within the top-down placement process. Section 3 discusses effective implementation of the quadratic placement methodology. We briefly review previous results [5] that suggest use of Krylov-subspace solvers, along with correlation convergence criteria within the solvers, to improve efficiency. We then focus on the coupling between the linear system solver and the min-cut partitioning step as the key to implementing quadratic placement: issues include the type of wirelength-based objective addressed by the solver, how the solver result is used by the partitioner, and the type of partitioning engine employed. In Section 4, we use a high- quality placement testbed to experimentally assess our various hypotheses. Our most significant results show that the solver-partitioner coupling may have historically been motivated by the pre1990's weakness of min-cut parameters, i.e., numerical engines were needed to provide helpful hints to weak min-cut partitioners. In particular, we show that a modern multilevel FM implementation [2] derives no benefit from such coupling. We also contrast the abilities of linear-wirelength and squared-wirelength solver objectives to drive partitioners to good solutions. Finally, we show that insights obtained from study of individual min-cut partitioning instances (within top-down placement) apply to the overall top-down placement results as well. We conclude the paper in Section 5 with a list of ongoing research directions, and some comments on the relevance of the quadratic placement methodology to future design methodology requirements.

\section{A SYNTHESIS OF THE QUADRATIC PLACEMENT METHODOLOGY}

\subsection{Notation and Definitions}

A gate-level netlist is represented for placement by a weighted hypergraph. The $n$ vertices correspond to modules, with vertex weights representing module areas or routing requirements. Hyperedges correspond to signal nets, with hyperedge weights representing criticalities and/or multiplicities. The two-dimensional layout region is represented as an array of legal placement locations. Placement seeks to assign all modules of the design to legal locations, such that no modules overlap and chip timing and routability are optimized. The placement problem is a type of NP-hard quadratic assignment.

The numerical techniques used within the quadratic placement methodology apply only to graphs (hyper-graphs with all hyperedge sizes equal to 2). Therefore, we must assume some transformation of hypergraphs to graphs via a net model. Throughout 
the discussion and experiments reported below, we use the standard clique model for nets of degree 10 or less, and the directed star model for nets of degree larger than 10 , to preserve sparsity. For a given multipin signal net with $k$ pins, the graph edges that represent the net may be constructed in several ways, e.g., a directed star model of $k-1$ edges, an unoriented star model, or an unoriented clique model of $(k(k-1) / 2)$ edges (see [3] for a review). The resulting weighted graph representation $G=$ $(V, E)$ of the circuit topology has edge weights $a_{i j}$ derived by "superposing" all derived edges in the obvious manner. The standard undirected clique model [25] assigns all clique edges weight $(1 /(k-1)){ }^{1}$

Definition The $n \times n$ Laplacian matrix $\mathbf{Q}=\left(q_{i j}\right)$ has entry $q_{i j}$ equal to $-a_{i j}$ for $i \neq j$ and diagonal entry $q_{i i}$ equal to $\sum_{j=1}^{n} a_{i j}$, i.e., the sum of edge weights incident to vertex $v_{i}$.

Certain vertices are fixed, typically due to preplacement of $\mathrm{I} / \mathrm{O}$ pads or the inducing of terminals around a block's periphery during top-down placement. All other vertices are movable. The one-dimensional placement problem seeks to place movable vertices onto the real line so as to minimize an objective function that depends on the edge weights and the vertex coordinates. The $n$-dimensional placement vector $\mathbf{x}=\left(x_{i}\right)$ denotes the physical locations of modules $v_{1}, \ldots, v_{n}$ on the real line, i.e., $x_{i}$ is the coordinate of vertex $v_{i}$. Let $c$ be the number of movable modules and let $f=n-c$ be the number of fixed ("pad") modules. Without loss of generality, the $c$ movable modules are $v_{1}, \ldots, v_{c}$ and the $f$ fixed modules are $v_{c+1}, \ldots, v_{n}$. The modules can always be permuted prior to optimization to ensure this condition is satisfied.
Squared Wirelength Formulation Minimize the objective

$$
\begin{aligned}
& \Phi_{q}(\mathbf{x})=\sum_{i>j} a_{i j}\left(x_{i}-x_{j}\right)^{2} \text { such that } \\
& x_{c+1}, \ldots, x_{n} \text { are fixed. }
\end{aligned}
$$

This function can also be written as

$$
\Phi_{q}(\mathbf{x})=(1 / 2) \mathbf{x}^{T} \mathbf{Q} \mathbf{x}
$$

We are interested in quadratic placers that solve the two-dimensional placement problem with a topdown approach, i.e., one-dimensional placement in the horizontal direction is used to divide the netlist into left and right halves, after which onedimensional placement in the vertical direction is used to subdivide the netlist into quarters, $e^{2}$. $^{2}$

\subsection{Essential Structure of a Quadratic Placer}

We now state the essential components of the quadratic placement methodology. Our primary goal is to establish the coupling between (i) numerical methods for sparse linear systems and (ii) mincut optimizations or other means of "spreading", or "legalizing", a continuous placement solution. We illustrate our discussion by referring to the PROUD algorithm of Tsay et al. [32, 33].

Like other works, PROUD considers the squared wirelength objective $\Phi_{q}(\mathbf{x})=(1 / 2) \mathbf{x}^{T} \mathbf{Q x}$. An unconstrained formulation is obtained by optimizing the objective function $\Phi_{q}(\mathbf{x})$ for $c$ movable modules while satisfying $f$ fixed pad constraints, but without discrete slot constraints. (The term slot constraint, originated by Cheng and Kuh [9], refers

\footnotetext{
${ }^{1}$ Some other clique models have been proposed, e.g., the model of [33] assigns all clique edges weight $8 / k^{3}$. We have obtained similar results for the clique model of [33] as well as for a directed star model.

2"Decomposition" of the two-dimensional placement problem into independent one-dimensional placement problems is used in the quadratic placement methodology to yield smaller linear systems. Notice that for the quadratic objective, the Euclidean problem decomposes cleanly into coordinates (by Pythagoras' theorem) while the Manhattan problem does not. (Hence, we presumably are minimizing squared Euclidean wirelength.) For the linear objective, the Euclidean problem does not decompose while the Manhattan objective does.
} 
to the fact that a legal placement must locate modules within the two-dimensional array of allowed locations (slots). E.g., the first-order slot constraint forces the sum of module coordinates to equal the sum of slot coordinates.) The objective function can be written as

$$
\begin{aligned}
\Phi_{q}(\mathbf{x}) & =\frac{1}{2}\left[\mathbf{x}_{c} \mathbf{x}_{f}\right]\left[\begin{array}{ll}
\mathbf{Q}_{c c} & \mathbf{Q}_{c f} \\
\mathbf{Q}_{f c} & \mathbf{Q}_{f f}
\end{array}\right]\left[\begin{array}{c}
\mathbf{x}_{c} \\
\mathbf{x}_{f}
\end{array}\right] \\
= & \frac{1}{2}\left(\mathbf{x}_{c}^{T} \mathbf{Q}_{c c} \mathbf{x}_{c}+\mathbf{x}_{c}^{T} \mathbf{Q}_{c f} \mathbf{x}_{f}+\mathbf{x}_{f}^{T} \mathbf{Q}_{f c} \mathbf{x}_{c}+\mathbf{x}_{f}^{T} \mathbf{Q}_{f f} \mathbf{x}_{f}\right)
\end{aligned}
$$

where $\mathbf{x}_{f}$ denotes the vector of fixed module positions and $\mathbf{x}_{c}$ denotes the vector of movable module positions; the Laplacian $\mathbf{Q}$ is partitioned into four corresponding parts $\mathbf{Q}_{c c}, \mathbf{Q}_{c f}, \mathbf{Q}_{f c}$ and $\mathbf{Q}_{f f}$ with $\mathbf{Q}_{c f}^{T}=\mathbf{Q}_{f c}$.

In this formulation, the optimal positions of all movable modules are inside the convex hull of the fixed module locations [33]. Hence, we can consider the minimization problem for $\Phi_{q}(\mathbf{x})$ over this convex hull. Since $\Phi_{q}(\mathbf{x})$ is a strictly convex smooth function over a compact set (in $c$-dimensional Euclidean space), the unique minimum objective function value is attained at the extremal or a boundary point; the nature of the problem implies that it will be at the extremal point. To find the zero of the derivative of the objective function $\Phi_{q}(\mathbf{x})$, we solve the $c \times c$ linear system

$$
\nabla \Phi_{q}(\mathbf{x})=\mathbf{Q}_{c c} \mathbf{x}_{c}+\mathbf{Q}_{c f} \mathbf{x}_{f}=0
$$

which can be rewritten as

$$
\mathbf{Q}_{c c} \mathbf{x}_{c}=-\mathbf{Q}_{c f} \mathbf{x}_{f}
$$

This development is similar to that of other "force-directed" or "resistive network" analogies (see, e.g. [36, 27, 14,9]. The essential tradeoff is the relaxation of discrete slot constraints, along with the changing of the "true" linear wirelength objective into a squared wirelength objective, to obtain a continuous quadratic objective for which a global minimum can be found. The typical resulting "global placement" concentrates modules in the center of the layout region. Hence, the key issue is how the "global placement" (actually, a "continuous solution obtained using an incorrect objective") should be "spread" or "legalized" into a solution to the original discrete problem.

Two approaches have been used to obtain a feasible placement from a "global placement". The first approach is based on assignment, either in one step (to the entire two-dimensional array of slots) or in two steps (to rows, and then to slots within rows) [14]. The second and more widely-used approach is based on partitioning: the global placement result is used to derive a horizontal or vertical cut in the layout, and the continuous squaredwavelength optimization is recursively applied to the resulting subproblems (see $[36,27,9,24]$ ). The main difficulty is making partitioning decisions on the extremely overlapped modules in the middle of the layout. The obvious median-based partitioning (find the median module and use it as a "splitter") is sensitive to numerical convergence criteria. Thus, FM-type iterative improvement strategies $([22,13]$; see [3] as well as the discussion of Section 3 for a review) are commonly used to refine the resulting partitioning (see, e.g. [24]). Since the typical objective for iterative improvement partitioning is some form of minimum weighted cut, ${ }^{3}$ the quadratic placement methodology can be seen to be quite similar in structure to top-down min-cut

\footnotetext{
${ }^{3}$ Formally, denote the $n$ modules of the netlist hypergraph $H(V, E)$ as $V=\left\{v_{1}, v_{2}, \ldots, v_{n}\right\}$. A net $e \in E$ is a subset of $V$ with size greater than one. A bipartitioning $P=\{X, Y\}$ is a pair of disjoint clusters (i.e., subsets of $V) X$ and $Y$ such that $X \cup Y=V$. The cut of a bipartitioning $P=\{X, Y\}$ is the number of nets which contain modules in both $X$ and $Y$, i.e., $\operatorname{cut}(P)=|\{e \mid e \cap X \neq \emptyset, e \cap Y \neq \emptyset\}|$. Let $A(v)$ denote the area of $v \in V$ and let $A(S)=\Sigma_{v \in S} A(v)$ denote the area of a subset $S \subseteq V$. Given a balance tolerance $r$, the min-cut bipartitioning problem seeks a solution $P=\{X, Y\}$ that minimizes cut $(P)$ subject to $(A(V)(1-r) / 2) \leq A(X), A(Y) \leq(A(V)(1+r) / 2)$.
} 
placers, with initial cuts induced from (one-dimensional) placements under the squared-wirelength objective. $^{4}$

We summarize the essential structure of a quadratic placer as consisting of:

- a sparse linear systems solver;

- a min-cut iterative (FM-type) partitioner; and

- a top-down hierarchical min-cut framework wherein for any given partitioning instance the solver results are used to induce an initial solution for the iterative partitioner.

\section{EFFECTIVE IMPLEMENTATION OF THE QUADRATIC PLACEMENT METHODOLOGY}

In this section, we list five major degrees of freedom in the implementation of the quadratic placement methodology. For two of these degrees of freedom - the use of modern Krylov-subspace solvers with pre-conditioners, and correlation convergence criteria - we refer the reader to earlier work of [5]. The remaining three degrees of freedom - linear wirelength vs. squared-wirelength objectives, the solver-partitioner interface, and the use of modern (multilevel) FM partitioners - are the focus of detailed experiments in Section 4 below.

Of course, we realize that there are many other degrees of freedom in the implementation of a "quadratic placer", e.g., the number of partitioning trials made at a given level of the top-down placement, the integration of metaheuristics such as cycling and overlapping [19], etc. In our experience, tuning these degrees of freedom is an important activity, requiring substantial effort. However, we have chosen to concentrate on what we believe are the more fundamental issues for quadratic placement implementation. In particular, our line of investigation is motivated by the recent advances in algorithm technology for iterative partitioning. Our main question is: Do modern partitioners really require seeding from (one-dimensional placements computed by) numerical solvers? Our discussion sets the stage for experimental evidence showing that implementation choices for quadratic placement are dominated by the strengths of modern multilevel partitioners. We find that the "quadratic placement methodology" no longer benefits significantly from the use of linear systems solvers that minimize quadratic wirelength objectives.

\subsection{Use of Krylov-subspace Solvers with Preconditioners}

Recall that quadratic placement requires solving sparse linear systems with dimensions on the order of the number of movable modules in a given onedimensional placement instance. The time complexity of an iterative solver depends on both the cost of a single iteration (which is constant during the solution of a given system) and the number of

\footnotetext{
${ }^{4}$ The PROUD placer [33, 32], which we have cited as a prototype for the quadratic placement methodology, is more careful than previous works in how it applies partitioning during the top-down min-cut process. Suppose that a vertical cut has been made along some centerline, so that the left and right halves must each be split by horizontal cuts. PROUD applies a "block Gauss-Seidel" analogy, as follows. Modules in the left half are ordered vertically, followed by terminal propagation (projection) to the centerline. The projected terminals then influence the vertical ordering of the modules in the right half. The modules of the right half are then fixed and projected to the centerline, where they influence a new vertical ordering of the left half. Eventually, both orderings converge and can be split to induce subproblems. In this way, PROUD affords more global interaction between siblings in the hierarchy (see also the "cycling and overlapping" metaheuristics discussed in [19]).

More generally, we recognize (i) that intermediate steps involving assignment or transportation may also be used to derive hierarchical subproblems from the initial global placement; (ii) that while top-down bipartitioning is the standard approach, hierarchical subproblems may also be derived by top-down quadrisection; (iii) that iterative partitioners can use more sophisticated (placement-based) objectives than the traditional weighted min-cut, and (iv) that any number of metaheuristics can be used within the general top-down framework to improve routability, timing, etc. (such recent works as [34,19] exemplify such possibilities). Nonetheless, we have chosen to synthesize a quadratic placement methodology based on FM-type iterative bipartitioning: this is the cleanest (and most typical) framework for the solver-partitioner interaction in quadratic placement.
} 
iterations needed until iterates adequately approximate the true solution. The theory of iterative methods shows that the number of iterations needed to obtain a good approximation in norm depends on the spectrum of the matrix involved [15]. Hence, the idea of a preconditioner - a way to transform the original system to an equivalent one with "improved" spectrum. Because most implementations of preconditioners entail additional periteration cost, one must carefully examine the overall efficiency of solver/preconditioner combinations on particular classes of instances: more expensive iterations must be balanced against the number of iterations saved.

The work of [5] experimentally compares iteration counts and CPU times for various combinations of solvers and preconditioners when solving typical systems arising within the quadratic placement methodology. These studies were motivated by the observation that works such as [33] use the 1950's-era SOR iteration to solve the linear system in Eq. (1), despite many improved methods for solving sparse symmetric linear systems having been developed in recent years. (See [7] for pseudocodes of solvers and preconditioners, as well as a taxonomy of the types of systems to which these iterations apply; [15] gives theoretical analysis). ${ }^{5}$ The authors of [5] test comparable implementations of solver-preconditioner combinations using the PETSc library [6], and conclude that BiConjugate Gradient Stabilized (BiCGS) is among the best solvers. Though it does not guarantee convergence, BiCGS is good even for degenerate (not necessarily symmetric) matrices and provides more robust convergence than conjugate gradient (CG). For preconditioners, Incomplete LU-factorization and the Successive Over-relaxation family (including SSOR) are particularly successful. In verifying the superiority of BiCGS, performance of SOR and SSOR was evaluated with the best value of $\omega$ parameter, which was determined to be $\omega=1.95$ (for SOR/SSOR solvers) and $\omega=1.0$ (for SOR/ SSOR preconditioners) over a range of problem instances.

\subsection{Linear-wirelength vs. Squared-wirelength Objectives}

In Section 2.2 above, we saw that the continuous placement formulation with squared wirelength, objective has a unique optimum solution that can be found by solving a sparse linear system. However, while being convenient to work with, the quadratic objective does not correspond to any intuitive physical quantity. Compared to the linear objective, the quadratic objective more heavily weights long connections and less heavily weights short connections. To see this, consider the example of two wires with respective lengths 2 and 10 . According to the linear objective, the relative cost of the long versus the short wire is $10 / 2=5$, but according to the quadratic objective, the relative cost of the long versus the short wire is $100 / 4=$ 25 - i.e., the cost ratio of long to short wires is much higher under the quadratic objective.

Several works have suggested that the following linear wirelength objective is superior for placement:

Linear Wirelength Formulation Minimize the $\mathrm{Ob}-$ jective

$$
\begin{aligned}
& \Phi_{l}(\mathbf{x})=\sum_{i>j} a_{i j}\left|x_{i}-x_{j}\right| \text { such that } \\
& x_{c+1}, \ldots, x_{n} \text { are fixed. }
\end{aligned}
$$

Mahmoud et al. [26] and Sigl et al. [31] demonstrate the superiority of the linear wirelength

\footnotetext{
${ }^{5}$ Briefly, iterative methods for solving large systems of linear equations can be classified as stationary or non-stationary. Stationary methods include Jacobi, Gauss-Seidel, Successive Over-relaxation (SOR) and Symmetric Successive Over-relaxation (SSOR). They are older, easier to implement and computationally cheaper per iteration. Non-stationary methods include Conjugate Gradient (CG), Generalized Minimal Residual (GMRes) and numerous variations. These are relatively newer and harder to implement, but afford much faster convergence. Additional computational expense per iteration is normally justified by much smaller numbers of iterations. Solvers which provide smooth convergence can be also used as preconditioners. Direct solvers present a different source of preconditioners for iterative methods, with examples being incomplete Cholesky (ICC), LU-factorization and incomplete LUfactorization (ILU).
} 
objective for analog and row-based placement, and Riess et al. [30] show that a one-dimensional placement which minimizes linear wirelength can lead to excellent netlist bipartitioning results.

Optimizing the linear wirelength is less straightforward. For example, there can be multiple optimal solutions (consider a single movable module connected to two fixed pads by edges of equal weight - this module can be optimally placed anywhere between the two pads). The set of optimal placements is again closed and contained within the convex hull of fixed pad locations (see [33]). Thus, direct minimization of the linear wirelength objective can be achieved by linear programming, but this is usually computationally expensive. Consequently, most placers that address the linear wirelength objective find a solution by iteratively solving several quadratic formulations. We use the GORDIAN-L placer [31] to illustrate this technique. (Note that the set of constraints that GORDIAN-L can handle is more general than described here. In particular, GORDIAN-L can handle center-of gravity constraints whereby the coordinates for any subset of modules must be centered around a prescribed center. However, the technique described here for optimizing a linear objective by transforming it into a quadratic objective is independent of the types of constraints applied.)

The objective $\Phi_{l}$ can be rewritten as

$$
\Phi_{l}(\mathbf{x})=\sum_{i>j} a_{i j}\left|x_{i}-x_{j}\right|=\sum_{i>j} \frac{a_{i j}\left(x_{i}-x_{j}\right)^{2}}{\left|x_{i}-x_{j}\right|} .
$$

If the $\left|x_{i}-x_{j}\right|$ term in the denominator were constant, then a quadratic objective would result which can be solved via the above technique. GORDIAN-L first solves the system $\Phi_{q}(\mathbf{x})$ to obtain a reasonable approximation for the $\left|x_{i}-x_{j}\right|$ terms. Call this solution $\mathbf{x}^{0}$. GORDIAN-L then derives successively improved solutions $\mathbf{x}^{1}, \mathbf{x}^{2}, \ldots$ until there is no significant difference between $x^{k-1}$ and $\mathbf{x}^{k}$. From a given solution $\mathbf{x}^{k-1}$, the next solution $\mathbf{x}^{k}$ is obtained by minimizing

$$
\begin{aligned}
\Phi_{l}^{k}\left(\mathbf{x}^{k}\right) & =\sum_{i>j} \frac{a_{i j}\left(x_{i}^{k}-x_{j}^{k}\right)^{2}}{\left|x_{i}^{k-1}-x_{j}^{k-1}\right|} \\
& =\sum_{(i, j) \in E} g_{i j}^{k} \cdot\left(x_{i}^{k}-x_{j}^{k}\right)^{2}
\end{aligned}
$$

where $g_{i j}^{k}=a_{i j} /\left|x_{i}^{k-1}-x_{j}^{k-1}\right|$. Note that the coefficients $g_{i j}^{k}$ are adjusted between iterations. The iterations terminate when the factors $\left(x_{i}^{k}-x_{j}^{k}\right)$ no longer change significantly. (It turns out that this approach is actually a special case of a method due to Weiszfeld [35]; see [5] for a detailed discussion.) Below, we will experimentally study the effects of the choosing the linear-wirelength, vs. the squared-wirelength, objective for one-dimensional placement within the quadratic placement methodology. In particular, we will consider the effects on individual cutsizes as well as on total placement wirelength.

\subsection{Correlation Convergence Criteria}

Any iterative solver builds a sequence of iterates that converges to the solution $\mathbf{x}$ of Eq. (1). In the quadratic placement methodology that we have described, the one-dimensional placement information is used to "seed", i.e., construct an initial solution for, the partitioner. How soon the iteration can be stopped will affect the CPU efficiency of the overall implementation. Typically, iterative solvers have stopping criteria, or convergence tests, that are based on some norm of the residual vector for an iterate, ${ }^{6}$ which is taken to represent error with respect to the true solution. In practice, most norms are equivalent, and various heuristics (check convergence every $j$ iterations, check differences of iterates rather than residual vectors, etc.) can reduce the time spent on convergence tests.

Constructing an initial min-cut partitioning solution from one-dimensional placement solution wastes information, particularly if nothing is retained but memberships of vertices in "left" and "right" initial partitions. If the final iterate will be sorted and split to induce an initial solution for

${ }^{6}$ When solving the system $\mathbf{A x}=\mathbf{b}$, the residual vector for a given iterate $\mathbf{x}^{k}$ is $\mathbf{b}-\mathbf{A} \mathbf{x}^{k}$. 
the min-cut partitioner, then the iteration should terminate as soon as further changes will be inessential to the partitioner. ${ }^{7}$ Determination of "inessential" fundamentally depends on the strength and stability of the partitioner, as will be discussed in the next subsection. However, regardless of what partitioner is used, solver iterations should certainly stop when the left and right groups stabilize. The work of [5] proposed a number of correlation convergence criteria, based on permutations, that may be useful in efficiently measuring such stabilization. Instead of residual norms, correlation convergence criteria use correlations and rank correlations between successive iterates to compute their similarity. Convergence is detected when such a measure becomes sufficiently close to 1 , and iterations are stopped. (Note the analogy to residual norms which are used in traditional convergence criteria, but tend to 0 rather than to 1.) In Section 4 below, we provide evidence that simple and efficiently-computed measures of correlation or rank correlation between successive iterates indeed yield useful correlation convergence criteria.

\subsection{The Solver-partitioner Interface}

A fourth degree of freedom in implementing the quadratic placement methodology is the "solverpartitioner interface", namely, the manner in which an initial solution for min-cut partitioning is constructed from a given solver iterate. The key decision concerns how much information to retain from the iterate when "seeding" the partitioner. Above, we noted that the usual practice is to sort coordinates of the iterate, then pre-seed some percentage of modules (corresponding to the most extreme coordinates) into the left and right initial partitions. Many implementation choices must be faced, e.g., whether the pre-seeded modules should be locked or unlocked within the partitioner; how to construct initial assignments for the remaining (not pre-seeded) modules; whether the pre-seeding should be based on module areas or module cardinalities; etc. In our experiments below, we apply the following procedure to create the initial bipartitioning solution.

- The midpoint of the iterate is determined, such that the sums of module areas on either side of the midpoint are as close to equal as possible.

- Fixed pads are assigned to left or right partitions according to whether they are to the left or right of the midpoint in the iterate.

- On the left (right) side of the midpoint, a prescribed seeding percentage of the modules with smallest (largest) coordinates are pre-seeded (but not locked) into the left (right) partition. The percentage is computed on each side according to module cardinality, rather than module area. In the experiments below, we study seeding percentages of $0 \%, 25 \%, 50 \%$ and $100 \%$.

- Remaining (not pre-seeded, not pad) modules are randomly assigned to the left and right partitions, such that the resulting partitioning is balanced. More precisely, we randomly order these remaining modules, then assign each in turn to the partition that currently has smaller total module area.

\subsection{Use of Modern (Multilevel) FM Partitioners}

Recall that a motivation of our present investigation is that the use of numerical linear systems solvers that minimize quadratic wirelength may be a historical accident, resulting from the pre-1990's weakness of min-cut partitioners. The standard FM bipartitioning approach consists of iterative improvement based on the Kernighan-Lin algorithm, using the improvement of Fiduccia-Mattheyses [13]. The FM algorithm begins with some initial solution $\{X, Y\}$ and proceeds in a series of passes. During a pass, modules are successively moved between $X$ and $Y$ until each module has been moved exactly once. Given a current solution $\left\{X^{\prime}, Y^{\prime}\right\}$, the

\footnotetext{
${ }^{7}$ This precept also applies when the iterate is used to "seed" the partitioner. For example, one can seed the initial partitioning solution with only a percentage (say, $20 \%$ ) of vertices having the most extreme coordinates (with all other vertices randomly assigned), because these vertices are more likely to be "correctly" assigned. The GORDIAN and GORDIAN-L placers use such a strategy $[24,31]$.
} 
previously unmoved module $v \in X^{\prime}$ (or $Y^{\prime}$ ) with highest gain $\left(=\operatorname{cut}\left(\left\{X^{\prime}-v, Y^{\prime}+v\right\}\right)-\operatorname{cut}(\{X, Y\})\right)$ is moved from $X^{\prime}$ to $Y^{\prime}$. After each pass, the best solution $\left\{X^{\prime}, Y^{\prime}\right\}$ observed during the pass becomes the initial solution for a new pass, and the passes terminate when a pass does not improve the initial solution.

Recent work [1,2,17, 18, 20,21] has illustrated the promise of multilevel approaches for partitioning large circuits. Multilevel partitioning recursively clusters ("coarsens") the instance until its size is smaller that a given threshold, then unclusters ("uncoarsens") the instance while applying a partitioning refinement algorithm.Work in multilevel partitioning was originally prominent in the scientific computing literature for partitioning finite-element graphs [18, 21, 28]. Hendrickson and Leland [18] developed a very efficient multilevel partitioning algorithm, included in their Chaco package. Metis, another multilevel partitioning package targeted to finite-element graphs, was developed by Karypis and Kumar [21]. In the VLSI CAD community, previous multilevel works include $[1,10,17$, 20] and [2]. As shown in [20] and [2], multilevel implementations of the FM approach give the strongest and most stable results yet reported in the VLSI partitioning literature. Thus, our fifth degree of freedom assesses the use of multilevel FM versus traditional FM implementations.

\section{EXPERIMENTAL RESULTS}

\subsection{Experimental Step}

Our top-down placement testbed includes the following elements.

- Plain FM and multilevel FM bipartitioning engines. The plain FM implementation uses a LIFO gain bucket organization for improved performance [16]. The multilevel FM implementation uses a CLIP-FM core [11] and follows the description of [2] with respect to use of heavy-edge matching for coarsening [20,2] and the value of the matching ratio $(r=0.33)$ for coarsening/uncoarsening.
- Numerical iterations to minimize the squaredwirelength and linear-wirelength objectives. To minimize squared wirelength, we use a BiConjugate Gradient Stabilized (BiCGS) solver without preconditioner, following the conclusions of [5]. To minimize linear wirelength, we apply the Weiszfeld iteration described in [4], using the same BiCGS solver. For this objective, we also use an ILU preconditioner since linear-wirelength minimization is inherently harder than squared-wirelength minimization.

- A top-down quadratic placer framework. Within this framework, relevant implementation choices are:

- Final (non-overlapping) module placements are evaluated by the sum of net bounding box half-perimeters.

- Nets are modeled as weighted graph edges for the numerical solvers using the standard clique model for nets of degree 10 or less, and the directed star model for nets of degree greater than 10 .

- Pads (or block terminals) are kept fixed in the positions originally specified by the designer.

- For multi-pin nets with pins outside the current partitioning instance, straightforward terminal propagation is used.

We use for standard-cell test cases from industry, which we read in Cadence LEF/DEF 4.5 format (see Tab. I).

Our basic experiment explores the various degrees of freedom from the previous section, as follows.

- For each bipartitioning instance with $\geq 50$ modules we use a solver to obtain a one-dimensional

TABLE I Parameters of four standard-cell test cases from industry

\begin{tabular}{lccc}
\hline & \multicolumn{2}{c}{ Test cases } & \\
Test case & Pad cells & Core cells & Nets \\
\hline Case 1 & 1083 & 5840 & 7637 \\
Case 2 & 182 & 8829 & 11962 \\
Case 3 & 711 & 12146 & 10880 \\
Case 4 & 185 & 20392 & 25634 \\
\hline
\end{tabular}


placement minimizing either squared or linear wirelength. For smaller instances, we do not produce placements and instead use a random initial solution in the bipartitioning.

- We use the linear placement to pre-seed an initial bipartitioning solution, either fully $(100 \%)$, partially $(50 \%$ or $25 \%)$ or not at all $(0 \%)$.

- We use either LIFO FM (FM) or ML CLIP-FM (MLFM) to obtain a minimum-cut exact bisection (using exact module areas, with tolerance equal to the largest individual module area in the instance). Note that when MLFM is used, its coarsening phase is constrained by the preseeding. Pre-seeded modules are not allowed to be matched to modules pre-seeded in the opposite partition.

- Exhaustive enumeration of all possible placements is used for end-cases having 5 modules or fewer.

- Each minimum-cut bisection is the best result from 5 multi-starts, with randomization in the initial assignment of non-pad/non-seeded modules, and in the heavy-edge matching based coarsening stage of MLFM.

Runtimes for our placer on a $300 \mathrm{MHz}$ Sun Ultra-10 are given in Table II. We emphasize that a tuned implementation would be much more efficient, e.g., in practice solvers would not be run with such rigorous convergence criteria. For the top-level bipartition instances alone, the quadratic solver required 11 seconds (178 iterations) and 45

TABLE II Total CPU times for our placer $(300 \mathrm{MHz}$ Sun Ultra-10) on smallest and largest test cases, under various configurations, with $100 \%$ pre-seeding

\begin{tabular}{lccc}
\hline Partitioner & $\begin{array}{c}\text { Analytical } \\
\text { placer }\end{array}$ & Case 1 (sec) & Case 4 (sec) \\
\hline LIFO FM & Quadratic & 110 & 660 \\
& Linear (Weiszfeld) & 180 & 767 \\
ML CLIP-FM & Quadratic & 156 & 820 \\
& Linear (Weiszfeld) & 205 & 802 \\
\hline
\end{tabular}

seconds (154 iterations), for Case 1 and Case 4 respectively. The 5 starts of LIFO FM required 2 and 11 seconds, and the 5 starts of ML CLIP-FM (including all clustering operations) required 4 and 20 seconds, for Case 1 and Case 4 respectively.

\subsection{Experimental Data}

For each experiment configuration and test case, we examine both the final placement result as well as the results of the top-level bisection step. In the context of the top-level bisection, we save 20 different iterates of the squared-wirelength minimization (BiCGS engine) to pre-seed partitioners in separate experiments. These 20 iterates are chosen uniformly spaced in the interval between the first and final solver iterates (the stopping criterion is for successive iterates to differ by less than $1 \times 10^{-8}$ times the norm of the residual). For each of these iterates, each partitioning engine, and each level of pre-seeding, Figures 1 through 4 show the best cuts achieved in 5 random starts, averaged over 5 separate trials. Oscillations in the Figures, particularly for MLFM results, are due to the randomizations inherent in the experimental setup. For linear-wirelength minimization only a one iteration is available, as Weiszfeld typically converges in a single iteration. The best cutsizes obtained when this iterate is used for pre-seeding $(100 \%$, $50 \%, 25 \%$ and $0 \%$ pre-seeding) are given in the captions of each Figure.

Tables III through VI document the similarity of each iterate of the squared-wirelength minimization to the next iterate and to the final iterate, using correlation and rank correlation measures [29]. We additionally report the similarity of the resulting partitioning solutions to the solution achieved using the final iterate. Here, the similarity measure is Hamming distance, i.e., the minimum number of modules that must be moved to transform one solution into the other. ${ }^{8}$

\footnotetext{
${ }^{8}$ More precisely, we treat each bipartitioning solution as a $0-1$ vector, so the Hamming distance between two partitioning solutions is a measure of how dissimilar two solutions are. If $X=x_{i}$ and $Y=y_{i}$ are two bipartitioning solutions, their Hamming distance is $\sum_{i=1}^{n}\left|x_{i}-y_{i}\right|$. If this quantity is larger than $n / 2$ then the coordinates in $Y$ are flipped and the quantity is recomputed.
} 

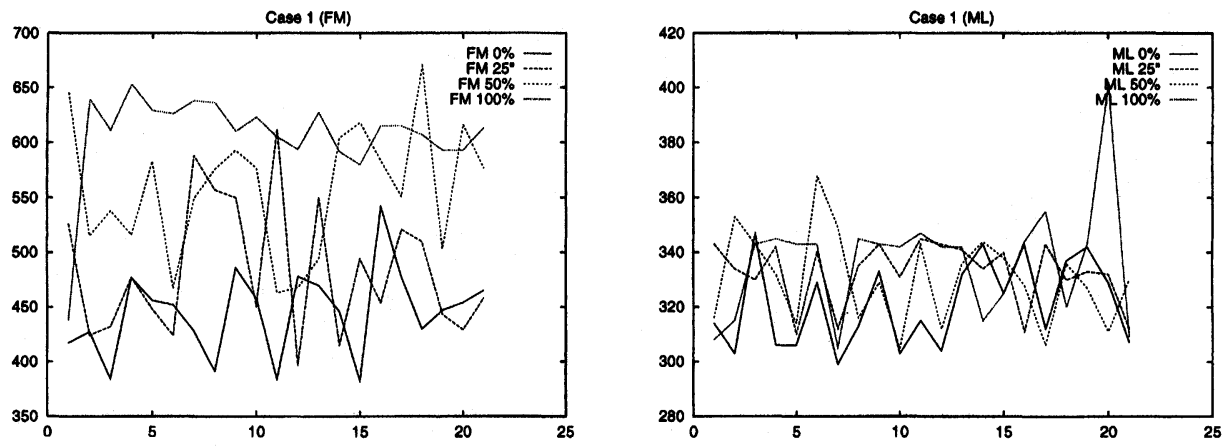

FIGURE 1 Best cut after pre-seeding with solver iterates for Case 1 . The $x$-axis is the index of the iterate, and the $y$-axis is the cutsize. When pre-seeding with the final (converged) Weiszfeld iterate, the best cuts were 485, 492, 550 and 571 for FM $(0 \%, 25 \%, 50 \%$ and $0 \%$ pre-seeding); and 315, 339, 328 and 307 for MLFM $(0 \%, 25 \%, 50 \%$ and $0 \%$ pre-seeding).
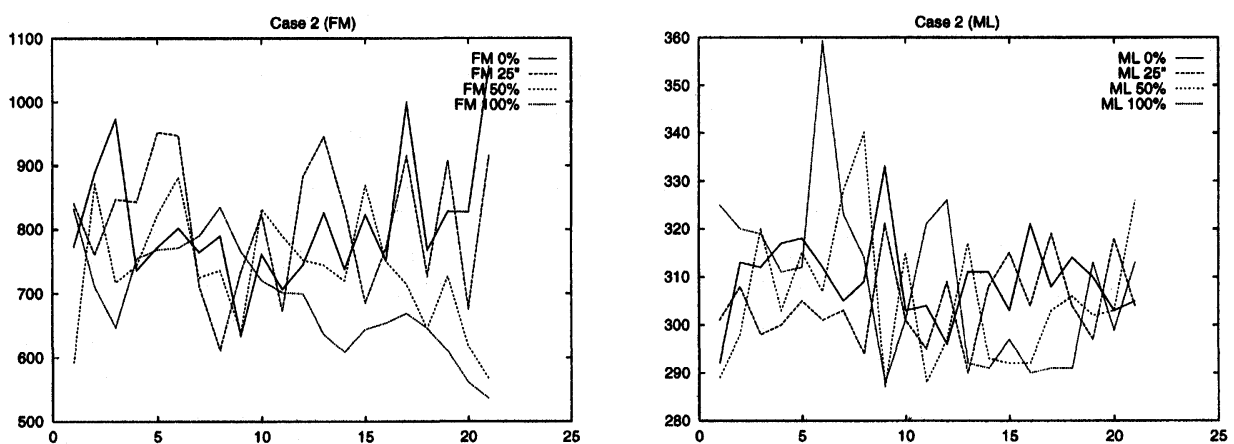

FIGURE 2 Best cut after pre-seeding with solver iterates for Case 2. The $x$-axis is the index of the iterate, and the $y$-axis is the cutsize. When pre-seeding with the final (converged) Weiszfeld iterate, the best cuts were 737, 745, 687 and 605 for FM $(0 \%, 25 \%, 50 \%$ and $100 \%$ pre-seeding); and $303,310,302$ and 315 for MLFM $(0 \%, 25 \%, 50 \%$ and $100 \%$ pre-seeding).
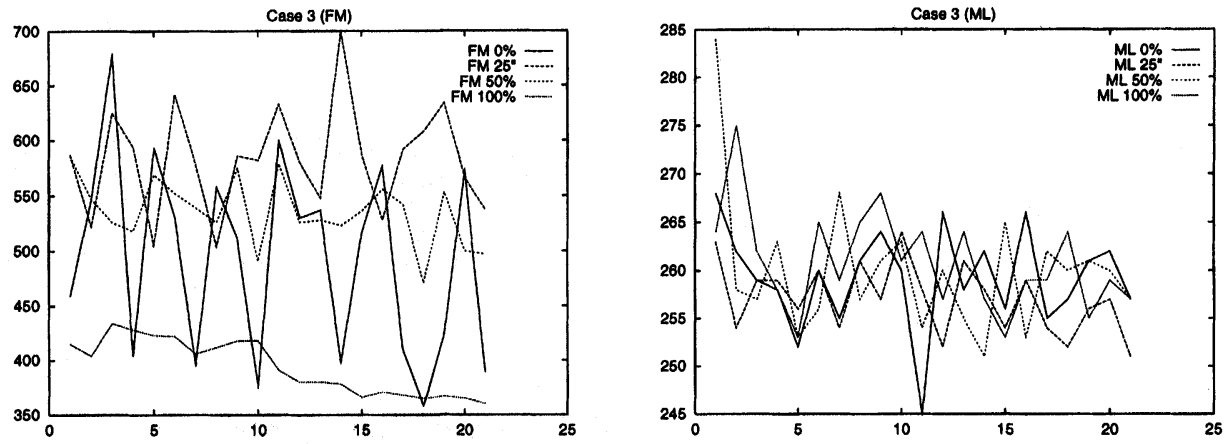

FIGURE 3 Best cut after pre-seeding with solver iterates for Case 3. The $x$-axis is the index of the iterate, and the $y$-axis is the cutsize. When pre-seeding with the final (converged) Weiszfeld iterate, the best cuts were 515, 492, 465 and 340 for FM $(0 \%, 25 \%, 50257,250,262$ and 251 for MLFM $(0 \%, 25 \%, 50 \%$ and $100 \%$ pre-seeding).

In our study of complete placement results, within each experimental configuration we preseed each partitioner call with the corresponding final solver iterate, and report the sum of net bounding box half-perimeters in the final placement. These results are given in Table VII. 

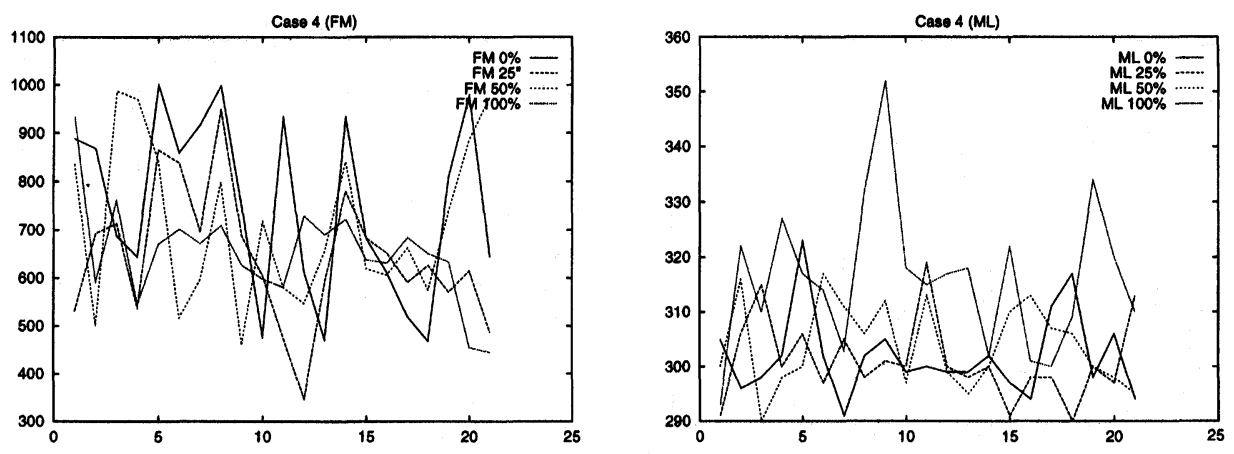

FIGURE 4 Best cut after pre-seeding with solver iterates for Case 4 . The $x$-axis is the index of the iterate, and the $y$-axis is the cutsize. When pre-seeding with the final (converged) Weiszfeld iterate, the best cuts were 737, 754, 643 and 590 for FM $(0 \%, 25 \%, 50 \%$ and $100 \%$ pre-seeding); and $318,328,337$ and 331 for MLFM $(0 \%, 25 \%, 50 \%$ and $100 \%$ pre-seeding).

TABLE III Correlation convergence studies for the top-level bisection of Case 1 and pre-seeding with early iterates of the squaredwirelength minimization

\begin{tabular}{|c|c|c|c|c|c|c|c|c|c|c|c|c|}
\hline \multirow[b]{4}{*}{ Iterate } & \multicolumn{12}{|c|}{ Case 1-quadratic wirelength } \\
\hline & \multirow[t]{3}{*}{ Corr. } & \multirow{3}{*}{$\begin{array}{l}\text { Rank } \\
\text { corr. } \\
\text { xt }\end{array}$} & \multirow[t]{3}{*}{ Corr. } & \multirow{3}{*}{$\begin{array}{l}\text { Rank. } \\
\text { corr. } \\
\text { nal }\end{array}$} & \multirow{2}{*}{\multicolumn{8}{|c|}{ Hamming distance to final }} \\
\hline & & & & & & & & & & & & \\
\hline & & & & & $0 \%$ & $25 \%$ & $50 \%$ & $100 \%$ & $0 \%$ & $25 \%$ & $50 \%$ & $100 \%$ \\
\hline 1 & 0.98974 & 0.938893 & 0.843959 & 0.811998 & 1220 & 1100 & 1514 & 2594 & 832 & 723 & 1447 & 1576 \\
\hline 2 & 0.998518 & 0.989313 & 0.887373 & 0.877041 & 1414 & 1170 & 1512 & 456 & 446 & 1069 & 1367 & 1543 \\
\hline 3 & 0.996944 & 0.98943 & 0.902439 & 0.893202 & 1193 & 1117 & 1426 & 555 & 750 & 802 & 1459 & 1590 \\
\hline 4 & 0.998996 & 0.992139 & 0.921435 & 0.909269 & 870 & 1264 & 1457 & 501 & 237 & 768 & 1179 & 980 \\
\hline 5 & 0.999269 & 0.997498 & 0.932654 & 0.924666 & 1233 & 1427 & 1139 & 217 & 992 & 149 & 1025 & 971 \\
\hline 6 & 0.998947 & 0.996191 & 0.941326 & 0.930699 & 1186 & 1276 & 1308 & 210 & 479 & 284 & 1443 & 984 \\
\hline 7 & 0.999457 & 0.997867 & 0.951809 & 0.939749 & 940 & 1331 & 1481 & 354 & 99 & 961 & 1464 & 1028 \\
\hline 8 & 0.999324 & 0.99767 & 0.958524 & 0.945523 & 1724 & 1189 & 1155 & 304 & 397 & 116 & 1447 & 937 \\
\hline 9 & 0.999328 & 0.998888 & 0.965899 & 0.951144 & 770 & 1089 & 1267 & 126 & 209 & 687 & 1529 & 923 \\
\hline 10 & 0.999833 & 0.999403 & 0.972098 & 0.954903 & 806 & 1480 & 1499 & 391 & 757 & 276 & 1065 & 1085 \\
\hline 11 & 0.999643 & 0.998387 & 0.975049 & 0.957211 & 1440 & 1253 & 1149 & 136 & 156 & 69 & 1452 & 191 \\
\hline 12 & 0.999698 & 0.998566 & 0.978848 & 0.96131 & 2065 & 1670 & 1157 & 155 & 546 & 606 & 1485 & 1627 \\
\hline 13 & 0.999885 & 0.999276 & 0.982403 & 0.965475 & 1442 & 1395 & 1326 & 422 & 604 & 714 & 1475 & 273 \\
\hline 14 & 0.99995 & 0.999491 & 0.98427 & 0.967885 & 1446 & 898 & 1133 & 213 & 997 & 91 & 1519 & 1692 \\
\hline 15 & 0.999777 & 0.997455 & 0.985507 & 0.96939 & 1391 & 1278 & 957 & 197 & 301 & 1045 & 1532 & 901 \\
\hline 16 & 0.9999 & 0.998801 & 0.988169 & 0.97446 & 1503 & 1368 & 1187 & 115 & 117 & 802 & 1437 & 216 \\
\hline 17 & 0.99981 & 0.996813 & 0.989733 & 0.97735 & 1404 & 1021 & 1090 & 115 & 334 & 499 & 1472 & 184 \\
\hline 18 & 0.999923 & 0.999515 & 0.991954 & 0.983116 & 1455 & 1372 & 1457 & 128 & 310 & 595 & 1101 & 1530 \\
\hline 19 & 0.999944 & 0.999332 & 0.99318 & 0.984812 & 1049 & 1074 & 1296 & 205 & 110 & 977 & 1593 & 1638 \\
\hline 20 & 0.999952 & 0.999312 & 0.994174 & 0.98658 & 1598 & 1109 & 1537 & 205 & 598 & 101 & 1129 & 1625 \\
\hline Final & - & - & 1 & 1 & 0 & 0 & 0 & 0 & 0 & 0 & 0 & 0 \\
\hline
\end{tabular}

\subsection{Discussion}

We first note that fully pre-seeded $(100 \%)$ runs are still somewhat randomized as we do not pre-seed partitioning instances of size 5 through 50 (small instances with 5 or fewer cells are solved optimally with an enumerative approach). Figures 1 through 4 justify the traditional quadratic placement methodology, in the sense that a (LIFO) FM partitioner clearly benefits from pre-seeding by a quadratic (squared-wirelength) solver. We see that full $(100 \%)$ pre-seeding reduces the FM cutsize by as much as $35 \%$, compared to no pre-seeding $(0 \%)$. On the other hand, MLFM cutsizes are clearly not improved, and in some cases are worse when pre-seeded with results from the quadratic 
TABLE IV Correlation convergence studies for the top-level bisection of Case $\mathbf{2}$ and pre-seeding with early iterates of the squaredwirelength minimization

\begin{tabular}{|c|c|c|c|c|c|c|c|c|c|c|c|c|}
\hline \multirow[b]{4}{*}{ Iterate } & \multicolumn{12}{|c|}{ Case 2-quadratic wirelength } \\
\hline & \multirow[t]{3}{*}{ Corr. } & \multirow{3}{*}{$\begin{array}{l}\text { Rank } \\
\text { corr. } \\
\text { xt }\end{array}$} & \multirow{3}{*}{\multicolumn{2}{|c|}{$\begin{array}{l}\text { Corr. Rank. } \\
\text { corr. } \\
\text { to final }\end{array}$}} & \multirow{2}{*}{\multicolumn{8}{|c|}{ LIFO FM $\begin{array}{ll}\text { Hamming distance to final } \\
\text { ML }\end{array}$}} \\
\hline & & & & & & & & & & & & \\
\hline & & & & & $0 \%$ & $25 \%$ & $50 \%$ & $100 \%$ & $0 \%$ & $25 \%$ & $50 \%$ & $100 \%$ \\
\hline 1 & 0.99445 & 0.705295 & \multicolumn{2}{|c|}{$0614096 \quad 0392017$} & 2378 & 2972 & 3731 & 4192 & 2367 & 299 & 2246 & 3861 \\
\hline 2 & 0.995601 & 0.960569 & 0.638045 & 0.682129 & 2548 & 2228 & 2570 & 2145 & 2832 & 3315 & 2384 & 2668 \\
\hline 3 & 0.998306 & 0.987488 & 0.659515 & 0.745051 & 2833 & 2165 & 2606 & 1449 & 278 & 791 & 2283 & 3895 \\
\hline 4 & 0.998757 & 0.992223 & 0.675138 & 0.773531 & 3471 & 3478 & 2690 & 1251 & 1750 & 4019 & 2204 & 3757 \\
\hline 5 & 0.998027 & 0.995468 & 0.689994 & 0.793538 & 3814 & 2470 & 2841 & 1311 & 1926 & 4402 & 917 & 3159 \\
\hline 6 & 0.998181 & 0.997799 & 0.710364 & 0.81365 & 3756 & 2398 & 3064 & 1261 & 1689 & 3407 & 1832 & 3825 \\
\hline 7 & 0.998545 & 0.998154 & 0.729696 & 0.826896 & 3752 & 2899 & 2291 & 1302 & 2710 & 339 & 2635 & 3864 \\
\hline 8 & 0.999051 & 0.999162 & 0.750016 & 0.842943 & 3110 & 3243 & 2521 & 1382 & 2519 & 4388 & 2355 & 3791 \\
\hline 9 & 0.999169 & 0.998872 & 0.7666 & 0.854092 & 2904 & 2628 & 2823 & 1219 & 2297 & 3307 & 2607 & 3847 \\
\hline 10 & 0.999185 & 0.9993 & 0.785464 & 0.869296 & 2399 & 2743 & 2388 & 1028 & 2752 & 3866 & 2416 & 3829 \\
\hline 11 & 0.998488 & 0.998786 & 0.803803 & 0.881228 & 2507 & 2923 & 2714 & 968 & 2529 & 3803 & 1829 & 3916 \\
\hline 12 & 0.998938 & 0.999046 & 0.829433 & 0.897179 & 1906 & 3037 & 1883 & 1013 & 2434 & 402 & 2374 & 3616 \\
\hline 13 & 0.998435 & 0.998875 & 0.851125 & 0.910766 & 2717 & 2379 & 2637 & 894 & 1985 & 4338 & 994 & 3833 \\
\hline 14 & 0.998147 & 0.998721 & 0.87616 & 0.925172 & 2755 & 2721 & 2670 & 655 & 1656 & 3732 & 4062 & 1429 \\
\hline 15 & 0.997945 & 0.998286 & 0.901718 & 0.939449 & 3107 & 2961 & 2549 & 902 & 1650 & 4372 & 2516 & 3162 \\
\hline 16 & 0.997114 & 0.998252 & 0.925989 & 0.954149 & 2546 & 2510 & 1937 & 981 & 617 & 3846 & 2313 & 1387 \\
\hline 17 & 0.998156 & 0.998953 & 0.95073 & 0.96779 & 2459 & 2642 & 1862 & 688 & 523 & 4389 & 2429 & 1495 \\
\hline 18 & 0.998083 & 0.998727 & 0.967119 & 0.976922 & 2755 & 2307 & 1321 & 685 & 583 & 3264 & 3312 & 101 \\
\hline 19 & 0.998301 & 0.99869 & 0.980637 & 0.98533 & 3324 & 2662 & 1682 & 478 & 2702 & 4061 & 2318 & 1224 \\
\hline 20 & 0.999164 & 0.999046 & 0.999164 & 0.999046 & 2454 & 2606 & 2036 & 59 & 1744 & 3993 & 2737 & 1266 \\
\hline Final & - & - & 1 & 1 & 0 & 0 & 0 & 0 & 0 & 0 & 0 & 0 \\
\hline
\end{tabular}

TABLE V Correlation convergence studies for the top-level bisection of Case 3 and pre-seeding with early iterates of the squaredwirelength minimization

\begin{tabular}{|c|c|c|c|c|c|c|c|c|c|c|c|c|}
\hline \multirow[b]{4}{*}{ Iterate } & \multicolumn{12}{|c|}{ Case 3-quadratic wirelength } \\
\hline & \multirow{3}{*}{ Corr. } & \multirow{3}{*}{$\begin{array}{l}\text { Rank } \\
\text { corr. } \\
\text { xt }\end{array}$} & \multirow[t]{3}{*}{ Corr. } & \multirow{3}{*}{$\begin{array}{l}\text { Rank. } \\
\text { corr. } \\
\text { lal }\end{array}$} & \multirow{2}{*}{\multicolumn{8}{|c|}{ Hamming distance to final }} \\
\hline & & & & & & & & & & & & \\
\hline & & & & & $0 \%$ & $25 \%$ & $50 \%$ & $100 \%$ & $0 \%$ & $25 \%$ & $50 \%$ & $100 \%$ \\
\hline 1 & 0.977774 & 0.805804 & 0.607565 & 0.638514 & 3169 & 3114 & 3475 & 3871 & 3813 & 4266 & 1450 & 2905 \\
\hline 2 & 0.990913 & 0.979015 & 0.690443 & 0.830686 & 1851 & 3700 & 2971 & 3886 & 277 & 3879 & 2984 & 2469 \\
\hline 3 & 0.993253 & 0.995148 & 0.744298 & 0.875779 & 2723 & 3525 & 3683 & 3680 & 2838 & 2646 & 2838 & 1111 \\
\hline 4 & 0.995701 & 0.996973 & 0.789769 & 0.898059 & 2669 & 3334 & 2955 & 2882 & 258 & 3948 & 1429 & 4361 \\
\hline 5 & 0.997248 & 0.998161 & 0.825311 & 0.915763 & 2788 & 3745 & 3404 & 2546 & 453 & 4350 & 376 & 4414 \\
\hline 6 & 0.997744 & 0.998767 & 0.852773 & 0.929199 & 3120 & 2974 & 3619 & 3044 & 187 & 4004 & 1405 & 4376 \\
\hline 7 & 0.998409 & 0.999132 & 0.87671 & 0.940352 & 2868 & 3725 & 2866 & 3121 & 887 & 293 & 3486 & 791 \\
\hline 8 & 0.998692 & 0.999151 & 0.895874 & 0.94939 & 2716 & 3143 & 3631 & 3069 & 2735 & 3264 & 1453 & 4331 \\
\hline 9 & 0.998422 & 0.999177 & 0.912526 & 0.957789 & 2774 & 4148 & 3215 & 2682 & 3140 & 3955 & 1690 & 1224 \\
\hline 10 & 0.998945 & 0.999413 & 0.92911 & 0.96547 & 2405 & 3593 & 2976 & 2827 & 3954 & 329 & 2857 & 2616 \\
\hline 11 & 0.999375 & 0.999638 & 0.941674 & 0.971347 & 2508 & 3380 & 2999 & 2927 & 279 & 3923 & 1309 & 3004 \\
\hline 12 & 0.998969 & 0.999268 & 0.950603 & 0.975456 & 3610 & 3974 & 3658 & 2954 & 3151 & 4377 & 1052 & 4036 \\
\hline 13 & 0.99946 & 0.999728 & 0.961839 & 0.981017 & 2960 & 3512 & 3512 & 3155 & 4379 & 3844 & 3311 & 4170 \\
\hline 14 & 0.999278 & 0.999556 & 0.96884 & 0.984048 & 2417 & 4267 & 3679 & 3056 & 4039 & 2638 & 1727 & 253 \\
\hline 15 & 0.999619 & 0.999797 & 0.976438 & 0.98757 & 2775 & 3662 & 3285 & 2899 & 345 & 3765 & 1535 & 3960 \\
\hline 16 & 0.999688 & 0.999816 & 0.981134 & 0.98958 & 2939 & 2911 & 3852 & 2992 & 1570 & 2494 & 1254 & 4397 \\
\hline 17 & 0.999829 & 0.999903 & 0.985025 & 0.991328 & 3259 & 3432 & 4040 & 3157 & 3147 & 2953 & 1785 & 4400 \\
\hline 18 & 0.999607 & 0.99973 & 0.98757 & 0.992461 & 2073 & 3582 & 3606 & 3214 & 2702 & 4010 & 1400 & 260 \\
\hline 19 & 0.999868 & 0.999921 & 0.991186 & 0.994243 & 3657 & 3548 & 4144 & 3195 & 3139 & 2930 & 3185 & 4387 \\
\hline 20 & 0.992901 & 0.995008 & 0.992901 & 0.995008 & 3184 & 2973 & 3892 & 3230 & 2713 & 318 & 3067 & 3748 \\
\hline Final & - & - & 1 & 1 & 0 & 0 & 0 & 0 & 0 & 0 & 0 & 0 \\
\hline
\end{tabular}


TABLE VI Correlation convergence studies for the top-level bisection of Case 4 and pre-seeding with early iterates of the squaredwirelength minimization

\begin{tabular}{|c|c|c|c|c|c|c|c|c|c|c|c|c|}
\hline \multirow[b]{4}{*}{ Iterate } & \multicolumn{12}{|c|}{ Case 4-quadratic wirelength } \\
\hline & \multirow{2}{*}{ Corr. } & \multirow{3}{*}{$\begin{array}{l}\text { Rank } \\
\text { corr. } \\
\text { xt }\end{array}$} & & \multirow{3}{*}{$\begin{array}{l}\text { Rank. } \\
\text { Corr. } \\
\text { nal }\end{array}$} & \multirow{2}{*}{\multicolumn{8}{|c|}{ Hamming distance to final }} \\
\hline & & & & & & & & & & & & \\
\hline & to next & & & & $0 \%$ & $25 \%$ & $50 \%$ & $100 \%$ & $0 \%$ & $25 \%$ & $50 \%$ & $100 \%$ \\
\hline 1 & 0.982226 & 0.627657 & 0.449465 & 0.500442 & 10060 & 1694 & 2664 & 7230 & 3736 & 3491 & 274 & 3843 \\
\hline 2 & 0.997357 & 0.955058 & 0.501609 & 0.822398 & 4321 & 3456 & 4176 & 3972 & 1165 & 141 & 91 & 549 \\
\hline 3 & 0.996133 & 0.997686 & 0.528977 & 0.897615 & 3985 & 2479 & 4134 & 1951 & 3697 & 2972 & 3744 & 495 \\
\hline 4 & 0.998251 & 0.998819 & 0.565941 & 0.911946 & 6956 & 5965 & 3863 & 2073 & 672 & 147 & 3235 & 2387 \\
\hline 5 & 0.997302 & 0.99901 & 0.594907 & 0.922433 & 8704 & 3442 & 3410 & 3312 & 3799 & 128 & 197 & 492 \\
\hline 6 & 0.997553 & 0.99931 & 0.633184 & 0.932492 & 6606 & 4215 & 3142 & 3434 & 3721 & 186 & 3244 & 522 \\
\hline 7 & 0.997205 & 0.999182 & 0.670902 & 0.940816 & 4795 & 2242 & 3975 & 1379 & 1185 & 3472 & 321 & 525 \\
\hline 8 & 0.996913 & 0.999497 & 0.713008 & 0.949826 & 7036 & 3866 & 4573 & 1038 & 1050 & 118 & 307 & 673 \\
\hline 9 & 0.997377 & 0.999591 & 0.755451 & 0.95665 & 9381 & 4113 & 4587 & 818 & 3655 & 106 & 63 & 618 \\
\hline 10 & 0.997351 & 0.999652 & 0.793298 & 0.962594 & 7964 & 4774 & 2866 & 908 & 506 & 119 & 105 & 2360 \\
\hline 11 & 0.996165 & 0.999566 & 0.829006 & 0.967859 & 6906 & 3039 & 4214 & 891 & 3643 & 116 & 257 & 517 \\
\hline 12 & 0.997065 & 0.999662 & 0.868215 & 0.97342 & 7586 & 2797 & 4029 & 1819 & 3700 & 171 & 3237 & 499 \\
\hline 13 & 0.997577 & 0.999622 & 0.898789 & 0.977999 & 4857 & 5310 & 3685 & 1749 & 3669 & 3060 & 88 & 294 \\
\hline 14 & 0.998607 & 0.999793 & 0.92385 & 0.982489 & 4596 & 5907 & 3705 & 1696 & 3698 & 3643 & 2554 & 313 \\
\hline 15 & 0.998706 & 0.999715 & 0.94039 & 0.985462 & 7030 & 2051 & 4050 & 1044 & 259 & 98 & 3775 & 343 \\
\hline 16 & 0.998054 & 0.999573 & 0.954994 & 0.988675 & 6853 & 3956 & 3746 & 951 & 713 & 141 & 277 & 340 \\
\hline 17 & 0.998796 & 0.999746 & 0.970299 & 0.992146 & 5066 & 2175 & 3856 & 1446 & 3630 & 206 & 282 & 3348 \\
\hline 18 & 0.999387 & 0.999803 & 0.980137 & 0.994402 & 5102 & 2505 & 2538 & 1108 & 3696 & 137 & 190 & 2338 \\
\hline 19 & 0.999211 & 0.999756 & 0.986279 & 0.996104 & 4929 & 1803 & 4577 & 880 & 631 & 153 & 78 & 2388 \\
\hline 20 & 0.999047 & 0.999685 & 0.999047 & 0.999685 & 7567 & 3330 & 3763 & 657 & 1111 & 210 & 116 & 413 \\
\hline Final & - & - & 1 & 1 & 0 & 0 & 0 & 0 & 0 & 0 & 0 & 0 \\
\hline
\end{tabular}

TABLE VII Average final wirelength results for top-down placement of Case 4

\begin{tabular}{|c|c|c|c|c|c|}
\hline \multirow[b]{2}{*}{ Partitioner } & \multirow[b]{2}{*}{ Analytical placer } & \multicolumn{4}{|c|}{ Ave final WL for $\%$ seeded } \\
\hline & & $0 \%$ & $25 \%$ & $50 \%$ & $100 \%$ \\
\hline \multicolumn{6}{|c|}{ CASE 1} \\
\hline \multirow{2}{*}{ LIFO FM } & Quadratic & 2.461 & 2.580 & 2.699 & 2.871 \\
\hline & Linear (Weiszfeld) & 2.468 & 2.539 & 2.752 & 3.105 \\
\hline \multirow[t]{2}{*}{ ML CLIP-FM } & Quadratic & 2.329 & 2.365 & 2.395 & 2.443 \\
\hline & Linear (Weiszfeld) & 2.329 & 2.358 & 2.370 & 2.452 \\
\hline \multirow{2}{*}{ LIFO FM } & Quadratic & 7.463 & 7.535 & 7.114 & 6.390 \\
\hline & Linear (Weiszfeld) & 7.463 & 7.574 & 7.111 & 6.414 \\
\hline \multirow{2}{*}{ ML CLIP-FM } & Quadratic & 5.250 & 5.344 & 5.353 & 5.365 \\
\hline & Linear (Weiszfeld) & 5.250 & 5.219 & 5.235 & 5.345 \\
\hline \multirow{2}{*}{ LIFO FM } & Quadratic & 3.970 & 3.917 & 3.874 & 3.633 \\
\hline & Linear (Weiszfeld) & 3.970 & 3.976 & 3.853 & 4.129 \\
\hline \multirow{2}{*}{ ML CLIP-FM } & Quadratic & 3.139 & 3.172 & 3.168 & 3.233 \\
\hline & Linear (Weiszfeld) & 3.139 & 3.152 & 3.202 & 3.320 \\
\hline \multirow[t]{2}{*}{ LIFO FM } & Quadratic & 9.436 & 9.297 & 9.503 & 8.921 \\
\hline & Linear (Weiszfeld) & 9.436 & 8.575 & 8.724 & 8.523 \\
\hline \multirow[t]{2}{*}{ ML CLIP-FM } & Quadratic & 6.860 & 6.948 & 6.953 & 7.047 \\
\hline & Linear (Weiszfeld) & 6.860 & 6.859 & 6.767 & 6.878 \\
\hline
\end{tabular}

solver. For all test cases MLFM with $0 \%$ preseeding is clearly superior to FM with $100 \%$ pre-seeding. This confirms that with modern partitioners, pre-seeding from analytical placements only hurts solution quality.
With regard to the use of a linear-wirelength minimizer, we observe that the FM partitions are still generally better with more pre-seeding, but that the improvement versus pre-seeding with the quadratic solver is somewhat unpredictable (in 
two cases, FM results are distinctly worse when pre-seeded with the Weiszfeld solution). ${ }^{9}$ The MLFM partitioner is still hurt by pre-seeding, but the linear-wirelength pre-seeding is less damaging than the squared-wirelength pre-seeding. Again, our main conclusion is that a modern multilevel partitioner no longer requires pre-seeding by an analytical solver, particularly for large instances.

The Hamming distance studies in Tables III through VI show that pre-seeding with early iterates leads to partitioning solutions that are structurally similar to those achieved with later iterates. ${ }^{10}$ We also see that correlation and rank correlation to the next iterate increase steadily. Since using later iterates does not usually improve cutsize, this suggests that correlation convergence measures can be used for early termination of numerical solvers. Finally, we observe that linear-wirelength minima do not seem strongly correlated with squaredwirelength minima.

We conclude this section by discussing the total wirelength results for complete placements obtained with each of the experimental configurations. From Table VII, we see that conclusions obtained for individual bisection instances still apply to complete placements: (i) FM is weaker than MLFM; (ii) FM benefits from $100 \%$ pre-seeding (but not always from $25 \%$ pre-seeding) but MLFM does better with no pre-seeding than with pre-seeding from quadratic placement; and (iii) MLFM performs somewhat better when partially pre-seeded with the placements produced by the Weiszfeld algorithm, while FM does not benefit from such pre-seeding. Overall, the worst results were achieved by FM with no pre-seeding, with partial pre-seeding by a squared-wirelength solver, or with full preseeding by a linear-wirelength solver. The best results were achieved by MLFM with no pre-seeding or with linear-wirelength based pre-seeding.

\section{CONCLUSIONS AND FUTURES}

We have synthesized the motivations and structure for a generic "quadratic placement" methodology, and developed a testbed that allows exploration of several key implementation decisions. Our experiments compare different combinations of partitioners, analytical solvers, and pre-seeding strategies within the solver-partitioned interface. We observe that:

- Traditional pre-seeding with a quadratic (squared-wirelength) engine does not improve either cutsize or placement results if a strong partitioner (e.g., ML Clip-FM) is used.

- If pre-seeding is used, earlier iterates are often as good as later iterates, and correlation convergence tests based on correlation and rank correlation between iterates can save CPU time by detecting when the relative order of module locations stabilizes.

- Pre-seeding with a linear-wirelength engine may be useful if issues such as stability and reproducibility of solution structure are considered-but such a conclusion, if true, would require a more elaborate experimental design to demonstrate.

These observations suggest that with the transition from classic FM partitioners to modern multilevel partitioners, quadratic engines may no longer be necessary in top-down placement, and may even lead to a loss of solution quality when applied.

Our ongoing research encompasses the following areas.

- We are improving our placement testbed to enable "apples-to-apples" comparison with commercial tools. This entails building infrastructure for timing-driven layout (industry-standard timing models, timing constraints formats, delay calculation and static timing analysis algorithms,

\footnotetext{
${ }^{9}$ From the results of [30], we would expect superior performance from a Weiszfeld-seeded FM partitioner.

${ }^{10}$ The Hamming distances are occasionally surprisingly large, even though cutsizes are similar. We recognize that our experiments do not address other issues, notably (i) the number of multi-starts required for stable solution quality, and (ii) reproducibility of solution structure, that may yet reveal advantages of solver-based pre-seeding strategies. Since these issues move us into details metaheuristics within the top-down placement, we defer them to future research.
} 
etc.) as well as interfaces to leading routing engines. Out existing placement capability is extremely competitive for wirelength minimization, but routability analyses and legality-checking are inferior to those in commercial systems, making direct comparisons difficult.

- We are studying non-hierarchical alternatives for the interface between analytical solvers and the layout substrate. Several recent approaches, based on novel formulations for both solver and "legalizer", appear promising.

- Finally, several drivers suggest looking beyond the quadratic placement methodology. (1) Wellknown limits of quadratic placers include inability to naturally model path timing constraints, invariance of orderings to unequal horizontal and vertical routing resources, and the requirement of pre-placed pads to "anchor" the analytical placement. (2) Future top-down design methodologies will tend to have smaller randomlogic blocks in order to gain predictability; these may not be large enough for a quadratic placer to show its "global awareness" and runtime advantages. (3) The advent of block-based designs, with synthesized glue logic spread out over disconnected regions, may lead to a design planning-block building - assembly flow that is also ill-matched to quadratic placers. Thus, we must also seek new placement approaches that can be better suited to future placement contexts.

\section{References}

[1] Alpert, C. J., Hagen, L. W. and Kahng, A. B., "A Hybrid Multilevel/Genetic Approach for Circuit Paritioning", Proc. ACM SIGDA Physical Design Workshop, April 1996, pp. $100-105$.

[2] Alpert, C. J., Huang, D. J.-H. and Kahng, A. B., "Multilevel Circuit Partitioning", Proc. ACM/IEEE Design Automation Conference, June 1997, pp. 530-533.

[3] Alpert, C. J. and Kahng, A. B. (1995). "Recent Directions in Netlist Partitioning: A Survey", Integration, the VLSI Journal, 19(1-2), 1-81.

[4] Alpert, C. J., Chan, T., Huang, D. J., Kahng, A. B., Markov, I., Mulet, P. and Yan, K., "Faster Minimization of Linear Wirelength for Global Placement", Proc. ACM/ IEEE Intl. Symp. on Physical Design, Napa, April 1997, pp. $4-11$.

[5] Alpert, C. J., Chan, T., Huang, D. J.-H., Markov, I. and Yan, K., "Quadratic Placement Revisited", Proc. ACM/IEEE Design Automation Conference, June 1997, pp. $752-757$.
[6] Balay, S., Gropp, W., Curfman McInnes, L. and Smith, B., "PETSc 2.0 User's Manual", Argonne National Laboratory, $1995 \mathrm{http}: / /$ www.mcs.anl.gov/petsc/petsc.html

[7] Barrett, R., Berry, M., Chan, T. F. et al., "Templates for the Solution of Linear Systems: Building Blocks for Iterative Methods", SIAM Press 1994, http://netlib2. cs.utk.edu/linalg/html_templates/Templates.html

[8] Bui, T., Heigham, C., Jones, C. and Leighton, T., "Improving the Performance of the Kernighan-Lin and Simulated Annealing Graph Bisection Algorithms", Proc. ACM/ IEEE Design Automation Conference, June 1989, pp. 775-778.

[9] Cheng, C. K. and Kuh, E. S. (1984). "Module Placement Based on Resistive Network Optimization", IEEE Trans. on Computed Aided Design, 3, 218-225.

[10] Cong, J. and Smith, M'L., "A Parallel Bottom-Up Clustering Algorithm with Applications to Circuit Partitioning in VLSI Design", Proc. ACM/IEEE Design Automation Conference, June 1993, pp. 755-760.

[11] Dutt, S. and Deng, W., "VLSI Circuit Partitioning by Cluster-Removal Using Iterative Improvement Techniques", Proc. IEEE Intl. Conf. on Computer-Aided Design, November 1996, pp. 194-200.

[12] Elman, H. C. and Chernesky, M. P. (1993). "Ordering Effects on Relaxation Methods Applied to the Discrete One-Dimensional Convection-Diffusion Equation", SIAM J. Numer. Anal., 30(5), 1268-1290.

[13] Fiduccia, C. M. and Mattheyses, R. M., "A Linear-Time Heuristic for Improving Network Partitions", Proc. ACM/IEEE Design Automation Conference, June 1982, pp. $175-181$.

[14] Fukunaga, K., Yamada, S., Stone, H. S. and Kasai, T., "Placement of Circuit Modules Using a Graph Space Approach", Proc. ACM/IEEE Design Automation Conference, June 1983, pp. 465-471.

[15] Hackbush, W., Iterative Solution of Large Sparse Systems, Springer Verlag, 1994.

[16] Hagen, L. W., Huang, D. J.-H. and Kahng, A. B., "Quantified Suboptimality of VLSI Layout Heuristics", Proc. ACM/IEEE Design Automation Conference, June 1995, pp. 216-221.

[17] Hagen, L. W., Huang, D. J.-H. and Kahng, A. B., "On Implementation Choices for Iterative Improvement Partitioning Algorithms", Proc. European Design Automation Conferences, September 1995, pp. 144-149.

[18] Hauck, S. and Borriello, G., "An Evaluation of Bipartitioning Techniques", Proc 16th Conf. on Advanced Research in VLSI, 1995, pp. 383-402.

[19] Hendrickon, B. and Leland, R., "A Multilevel Algorithm for Partitioning Graphs", Proc. Supercomputing, 1995. see also Tech. Rep. SAND93-1301, Sandia National Laboratories, 1993.

[20] Huang, D. J.-H. and Kahng, A. B., "Partitioning-Based Standard-Cell Global Placement with an Exact Objective", Proc. ACM/IEEE Intl. Symp. on Physical Design, Napa, April 1997, pp. 18-25.

[21] Karypis, G., Aggarwal, R., Kumar, V. and Shekhar, S., "Multilevel Hypergraph Partitioning: Application is VLSI Domain", Proc. ACM/IEEE Design Automation Conference, June 1997, pp. 526-529.

[22] Karypis, G. and Kumar, V., "Multilevel Graph Partitioning Schemes", Banerjee, P. and Boca, P., Editors, Proc. Intl. Conf. on Parallel Processing, 1995, 3, 113-122.

[23] Kernighan, B. W. and Lin, S. (1970). "An Efficient Heuristic Procedure for Partitioning Graphs", Bell Syst. Tech. J., 49(2), 291-307. 
[24] Krishnamurthy, B. (1984). “An Improved Min-Cut Algorithm for Partitioning VLSI Networks", IEEE Trans. on Computers, 33(5), 438-446.

[25] Kleinhans, J., Sigl, G., Johannes, F. and Antreich, K. (1991). "GORDIAN; VLSI Placement by Quadratic Programming and Slicing Optimization", IEEE Trans. on Computer Aided Design, 10(3), 356-365.

[26] Lengauer, T., Combinatorial Algorithms for Integrated Circuit Layout, Wiley-Teubner, 1990.

[27] Mahmoud, I. I., Asakura, K., Nishibu, T. and Ohtsuki, T. (1994). "Experimental Appraisal of Linear and Quadratic Objective Functions Effect on Force Directed Method for Analog Placement", IEICE Transactions of Fundamentals of Electronics, Communications and Computer Sciences, E77-A(4), 719-725.

[28] Otten, R. H. J. M., "Automatic Floorplan Design", Proc. ACM/IEEE Design Automation Conference, June 1982, pp. $261-267$.

[29] Ponnusamy, R., Mansour, N., Chaudhary, A. and Fox, G. C. (1994). "Graph Contraction for Mapping Data on Parallel Computers: A Quality-Cost Tradeoff”, Scientific Programming, 3(1), 73-82.

[30] Press, W. H., Teukolsky, S. A., Vetterling, W. T. and Flannery, B. P., Numerical Recipes in C: The Art of Scientific Computing, 2nd edn., Cambridge: Cambridge University Press, 1992.

[31] Riess, B. M., Doll, K. and Johannes, F. M., "Partitioning Very Large Circuits Using Analytical Placement Techniques", Proc. ACM/IEEE Design Automation Conference, June 1994, pp. 646-651.

[32] Sigl, G., Doll, K. and Johannes, F., "Analytical Placement: A Linear or a Quadratic Objective Function?", Proc. ACM/IEEE Design Automation Conference, June 1991, pp. 427-432.

[33] Tsay, R. S., Kuh, E. and Hsu, C. P. (1988). "Proud: A Sea-Of-Gate Placement Algorithm", IEEE Design and Test of Computers, pp. 44-56.

[34] Tsay, R. S. and Kuh, E. (1991). "A Unified Approach to Partitioning and Placement", IEEE Trans. on Circuits and Systems, 38(5), 521-633.

[35] Vygen, J., "Algorithms for Large-Scale Flat Placement", Proc. ACM/IEEE Design Automation Conference, June 1997, pp. 746-751.

[36] Weiszfeld, E. (1937). "Sur le Point pour Lequel la Somme des Distances de $n$ Points Données est Minimum", Tôhoku Mathematics J., 43, 355-386.

[37] Wipfler, G. J., Wiesel, M. and Mlynski, D. A., "A Combined Force and Cut Algorithm for Hierarchical VLSI Layout, Proc. ACM/IEEE Design Automation Conference, June 1982, pp. 671-677.

\section{Authors' Biographies}

Charles J. Alpert received the Ph.D. degree in computer science at the University of California at Los Angeles in 1996. He currently is a Research Staff Member for the Computer Aided Design Group at the IBM Austin Research Laboratory in Austin, TX. His research interests include partitioning, placement, noise avoidance, timing optimization, and clock distribution. He received two
Best Paper Awards at the 1994 and 1995 ACM/ IEEE Design Automation Conferences. Dr. Alpert is a member of IEEE and the program committee for the International Symposium on Physical Design.

Andrew E. Caldwell received the B.S. degree in Computer Science from UCLA, and is currently a $\mathrm{Ph} . \mathrm{D}$. student in Computer Science at the School of Engineering Applied Science, UCLA. His research interests include VLSI layout design, and graph algorithms.

Tony F. Chan is currently chair of the mathematics department at the University of California, Los Angeles, where he has been a professor since 1986. Previously, he was on the faculty of the Computer Science Department at Yale University. His current research interests include the design of efficient computational algorithms for large scale scientific computing (e.g., multigrid and domain decomposition algorithms, iterative methods, Krylov subspace methods, and parallel algoritms), VLSI circuit placement and PDE methods for image processing. $\mathrm{He}$ is a member of SIAM, AMS, and IEEE and is on the editorial boards of several leading journals in computational mathematics, including the SIAM J. Sci. Comp. and Numer. Algs.

Dennis Huang (S'93-M'98) received the B.S. degree form the National Taiwan University, Taipei, Taiwan, R.O.C., the M.S. degree from State University of New York, Stony Brook in 1991, and $\mathrm{Ph} . \mathrm{D}$. degree from University of California, Los Angeles in 1997, all in Computer Science.

Since 1991, he was a Research Assistant in the Department of Computer Science, University of California, Los Angeles. In summer of 1995, he was with High Level Design Systems, Santa Clara, CA. In 1997, he was with the Design Planning Group, Synopsys, Mountain View, CA. Currently, he is with the Layout Product Division, Avant! Corporation, Fremont, CA. His research interests are in the areas of physical design on VLSI CAD, with emphasis on circuit partitioning, placement, and clock routing.

Andrew B. Kahng received the A.B. degree in applied mathematics (physics) from Harvard College, and the M.S. and Ph.D. degrees in computer 
science from the University of California at San Diego. He joined the computer science faculty at UCLA in July 1989, and is currently professor and vice-chair for graduate studies. From April 1996 through September 1997, he was on sabbatical leave and leave of absence from UCLA, as a Visiting Scientist at Cadence Design Systems, Inc. Professor Kahng has received NSF Research Initiation and Young Investigator awards, and a DAC Best Paper award. His research interests include VLSI physical layout design and performance analysis, combinatorial and graph algorithms, and stochastic global optimization.

Igor L. Markov graduated from Kiev University, Ukraine in 1993 and received his M.A. degree in pure mathematics from UCLA in 1994. He worked for parametric Technology Corporation in 1994 and is currently a doctorate student and in Computer science and research assistant at UCLA. $\mathrm{He}$ is a student member of AMS, ACM, IEEE and IEEE Computer Society. Igor Markov is currently working in several areas of VLSI physical layout design including hypergraph partitioning and placement. He is interested in hypergraph algorithms, analytical algorithms, continuous and combinatorial optimization.

Max S. Moroz is a Ph.D. student in decision sciences at the Anderson Graduate School of Management, UCLA. His research interests include modeling business processes, graph algorithms, and reusable software. He is a student member of IEEE and INFORMS. 

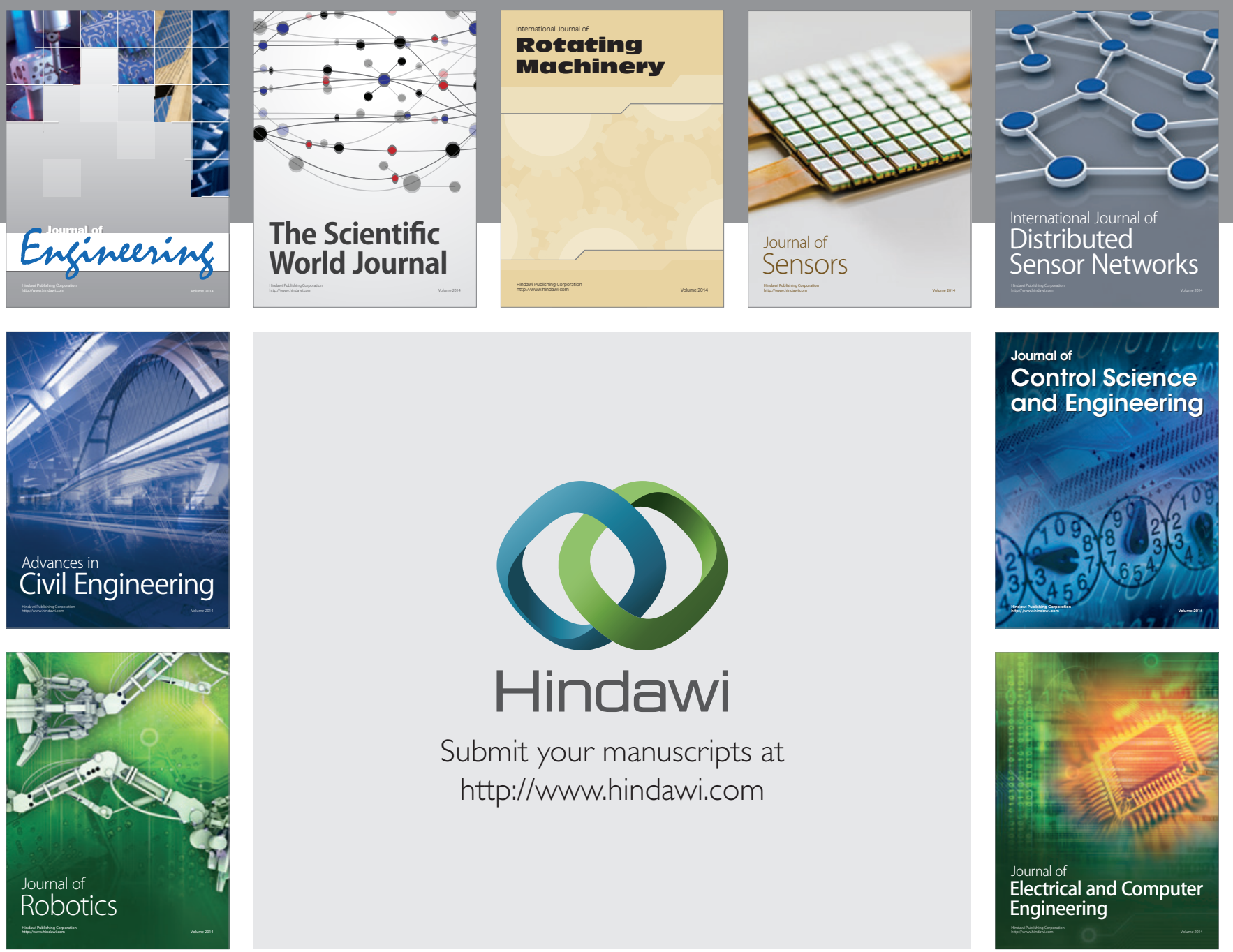

Submit your manuscripts at

http://www.hindawi.com
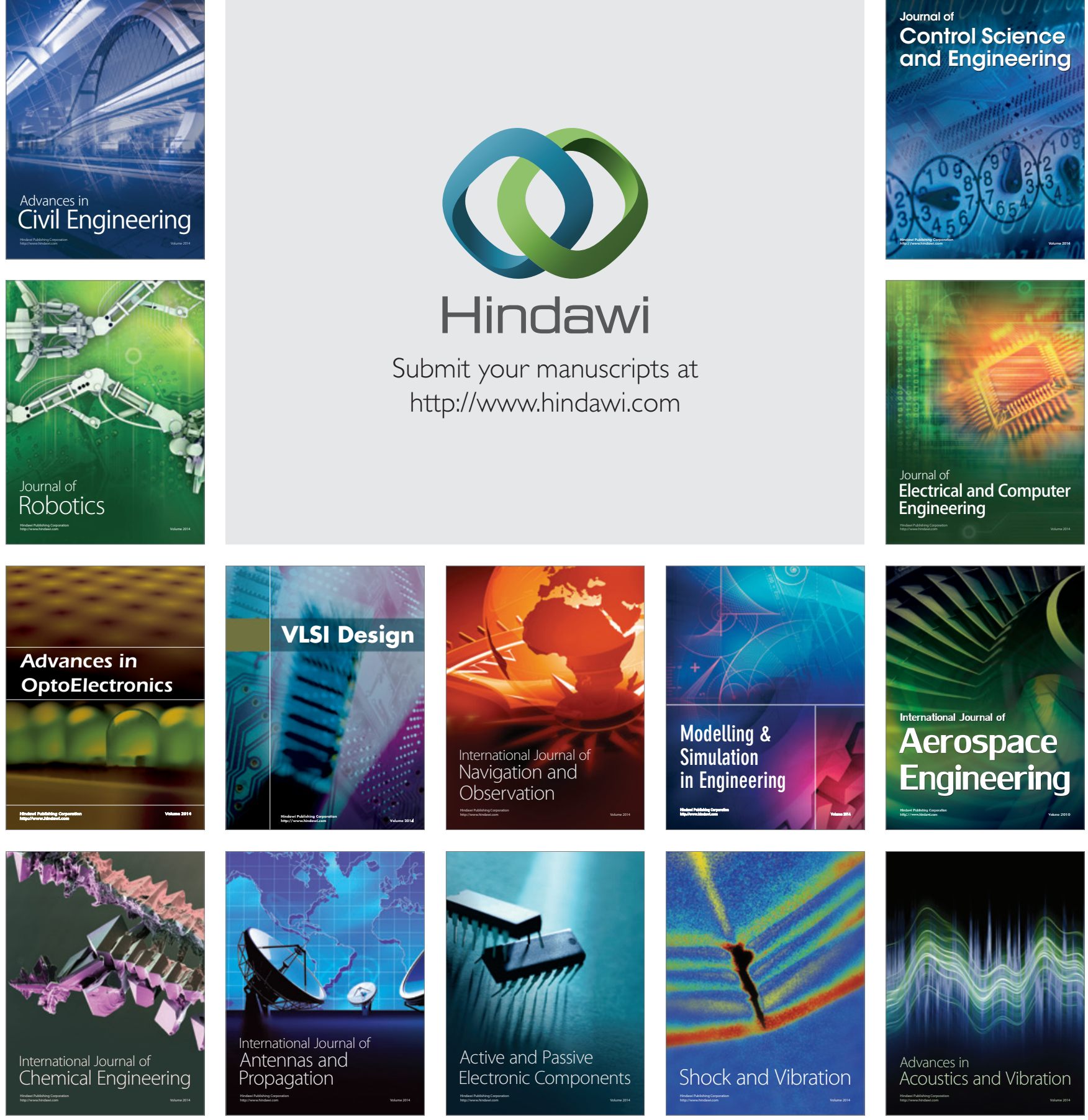